Review

\title{
The True Complexity of Photothermal Therapy: A Brief Perspective
}

\author{
Gabriel Alfranca ${ }^{1,2,3}$, Daxiang Cui ${ }^{2}$, Jesus Martinez de la Fuente ${ }^{1,2,3}$ \\ ${ }^{1}$ Institute of Nano Biomedicine and Engineering, Department of Instrument Science and Engineering, Shanghai Engineering Centre \\ for Intelligent Diagnosis and Treatment Instrument. Shanghai Jiao Tong University, Shanghai 200240, China. \\ ${ }^{2}$ Instituto de Ciencia de los Materiales de Aragón (ICMA-CSIC/University of Zaragoza) C/Pedro Cerbuna 12, 50009, Zaragoza, \\ Spain. \\ ${ }^{3}$ Centro de Investigación Biomédica en red en Bioingenieria Biomateriales y Nanomedicina (CIBER-BBN), Zaragoza, Spain. \\ C Corresponding author. E-mail: jmfuente@unizar.es
}

Received: May 23, 2017; Accepted: Jun. 5, 2017; Published: Jun. 8, 2017

Citation: Gabriel Alfranca, Daxiang Cui, and Jesus Martinez de la Fuente, The True Complexity of Photothermal Therapy: A Brief Perspective: A Survey. Nano Biomed. Eng., 2017, 9(2): 129-134.

DOI: $10.5101 /$ nbe.v9i1.p129-134.
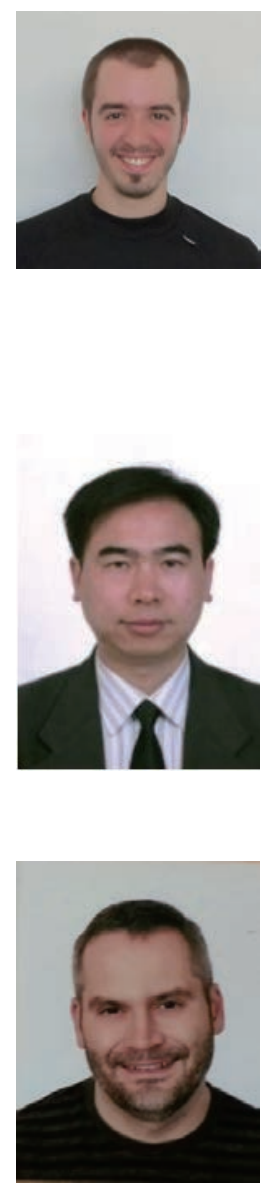

Gabriel Alfranca carried out his BD and MD at the University of Zaragoza (Spain), obtaining his degree in Biochemistry. During his MD’s project he entered the research group of Dr. de la Fuente and got a contract from the university that allowed him to stay until he started his double degree $\mathrm{PhD}$ studies in the same research group in conjunction with the research group of Prof. Cui in Shanghai Jiao Tong University, where he got a CSC scholarship type A. His research interests are the understanding of the interactions between nanomaterials and biological structures, especially during complex pathological processes such as cancer, and the development of nano-biosensors for ultra-low detection of biomolecules.

Prof. \& Dr. Daxiang Cui obtained his MD and PhD at the Fourth Military Medical University in 1998, was a post-doctoral fellow in Max Planck Institute for Metals Research from 2001 to 2004, and was a visiting professor in Waseda University from 2007 to 2008. So far he is Distinguished Professor, Changjiang Scholar at Shanghai Jiao Tong University. He has published over 280 papers in international peer-reviewed journals, high-index is 45 . His research interests include controlled synthesis and biosafety evaluation of nanomaterials, nanoparticles-labeling and nano-effects-based tumor theranostic technologies, high efficient drug delivery system and RNA nano-drug.

Prof. \& Dr. Jesus M de la Fuente finished his PhD work in 2003 at the Institute of Chemical Research from CSIC. Once he obtained his PhD, he moved to the Centre for Cell Engineering University of Glasgow (UK) during two years. In July 2005, he went back to the Institute of Chemical Research (Seville, Spain). In June 2007, Dr. de la Fuente established the Nanotherapy and Nanodiagnostic Group at the Institute of Nanoscience of Aragon (University of Zaragoza, Spain). Since 2014, he is a Permanent Researcher at the Spanish National Research Council- Aragon Materials Science Institute (Zaragoza, Spain). His research interests include the understanding the development and biological application of nanoparticles, nanobiosensors for ultra-low detection, drug delivery, vectorisation of nanoparticles, and new methodologies and therapeutic approaches for cancer diagnosis and treatment. 


\begin{abstract}
Despite its undoubted potential, recent studies have began to highlight the complex processes of photothermal therapy. In this perspective, we tackle several issues that affect current research on photothermal therapy, such as the influence of other factors produced in the process, for instance reactive oxygen species or vapor nanobubbles. After focusing on several of the aspects of photothermal therapy that need further clarification, we step back and see this therapy from a wider angle in order to identify its weaknesses. Finally, we briefly summarize what we consider should be the orientation and key focus of future researches in order to establish the full potential of this rapidly advancing and promising therapy.
\end{abstract}

Keywords: Plasmonic nanoparticles; Photothermal therapy; Near infra-red; Therapeutic window; Reactive oxygen species; Vapor nanobubbles

\section{Introduction: Hyperthermia, Nowadays}

Photo-thermal Therapy (PTT) is a clinical approach that has been developed extensively over the last few decades as an alternative or complementary approach to address certain pathological conditions such as cancer. Combined with nanoparticles, it represents a relatively novel and attractive tool that has grabbed the attention of both researchers and clinicians [1, 2]. It consists of the use of nanoparticles with the ultimate goal to specifically localize in the diseased area, which upon illumination with a laser are able to convert the incoming light into heat which ultimately induce cell death of the affected area [3], similar to a nanometric scalpel. One of the principle advantages of this approach is that the laser wavelength can be tuned to a range in which the absorption by biological structures is minimal, mainly within the near infra-red (NIR) range, the so called "therapeutic window" [4]; it goes without saying that for that purpose the nanoparticles need to be engineered to absorb irradiation at those wavelengths, and thus generate heat upon irradiation. In addition, the biodistribution and subcellular location are issues that must be deeply understood to prevent the nanoparticles to locate in an unspecific manner.

This technique, referred to as nanoparticle-mediated Photothermal therapy (NP-PTT), is a trending topic in the last years where numerous scientific publications have explored the possibilities of this new therapy, with special focus on the treatment of cancer. In such anti-cancer applications, the NP-PTT can be used as a treatment by itself or in combination with other techniques or therapeutic approaches in order to obtain adventitious synergistic effects. A clear example is in multi-drug resistance of tumors towards traditional treatments, nowadays considered as one of the major challenges for chemotherapy. Approaches using multi-functional nanoparticles are amongst the most important methodologies to address this issue [3]; however, there are still many questions that must be answered.

The enormous possibilities that this apparently simple concept has is reflected by the number of works published each year. Various types of starting materials have being used in order to synthesize the nanoparticles: gold, carbon, copper, molybdenum, tungsten, iron, palladium and conjugated polymers [1], both individually or combined. Among all those materials used, by far the most popular is gold, thanks to its straightforward synthesis, ease of functionalization, optical properties and low toxicity [3, 5]. Carbon-based nanomaterials also represent a strong branch of research thanks to its structural versatility, allowing to create configurations with unique optical properties such as carbon nanotubes and nanographene oxide [1], among others [5]. In addition, there have been many attempts of alternative approaches by combining PTT with other techniques like drug delivery [3, 6, 7], photodynamic therapy [3, 8], optoacoustic imaging $[9,10]$ and immunotherapy [11] among others. Many of the aforementioned techniques have being tested in vivo, revealing promising results and a low toxicity profile. However, despite all these efforts, only a few designs have reached pre-clinical trials, and their true therapeutic capability is yet to be revealed [1]. In summary, this promising therapy, despite its potential and very many applications in health, still requires a great deal of fundamental research to address certain issues that are still not fully understood.

\section{You Cannot Hit What You Cannot See: Understanding PTT}

All in all, the amount of effort seems to be focused 
in the development of different approaches to the same problem, instead of understanding the problem in detail. There are very few publications performing a meticulous study on how the different tissues react towards the presence of the nanosystems while being irradiated, other than their in vitro and in vivo toxicity; not to mention the lack of studies trying to elucidate the metabolic and signaling pathways that occur inside a cell during and after it is exposed to such unusual conditions of laser irradiation. It must be acknowledged that the way the cell reacts to the irradiation determines its fate, which can occur via a programmed apoptotic pathway, or towards the necrotic cell death mechanism, with all its pro-inflammatory consequences.

Besides, the way the nanoparticles react to the laser illumination is still not clear. Apart from the heat produced by their plasmonic oscillations, there are some studies that suggest that there are other processes involved, like the apparition of more unpredictable reactive oxygen species (ROS) $[8,12$, 13] as a result of the interaction of the nanomaterials with the incoming irradiation. ROS are highly reactive radicals (e.g. hydroxide, superoxide and singlet oxygen) naturally present in cells, produced primarily in the mitochondria, and also generated by external factors, such as incoming irradiation. If raised higher than basal levels, ROS are able to disrupt biological functions by altering proteins, biological membranes and nucleic acids, and thus induce off-target cell death in an uncontrolled manner [12]. ROS levels can increase during heat generation and PTT $[12,13]$, arising in unpredictable outcomes that may undermine the accuracy offered by this therapeutic approach. Minai and coworkers were amongst the first to publish a thorough study of the processes that induce ROS during PTT [13]. Other studies have described certain phenomena derived from the local heat at the proximity of the nanoparticles which temperature is much higher than the rest of the media, like the case of vapor nanobubbles (VNB) [14]. In this study, Xiong and co-workers mentioned that VNB had almost no heat transmission, and thus the disruption that they cause is due to a mechanical process that can alter the surroundings of the media where the nanoparticles are located. All these recently described by-products and uncontrolled effects derived from PTT not only reveal that this promising treatment is much more complex than what previously thought, but also that irradiation has a certain degree of unpredictability.

Although it is true that under heating conditions cell stress can ultimately derive on death, this is not the only concern. One important factor of the abovementioned unpredictability lies on the arduous control of the retention of nanoparticles inside a specific organelle, or the difficulty to generate a controlled diffusion of the nanostructures across the entire cell. At first glance it may seem that the heat generated can be retained in a single cell compartment at lower intensities or, if the irradiation is increased, it can literally "melt" the biological membranes [2], starting the diffusion of the nanoparticles throughout the cell [15] (Fig. 1). Following this statement, one could think that the control of this process could be addressed in a relatively straightforward way as long as the heat produced is the only actor, which unfortunately seems not to be the case.

It is well accepted that, apart from other effects, both ROS and temperature increase are able to facilitate the rupture or fluidity of the cellular membranes ${ }^{2}$, facilitating the entrance of the nanostructures through the various cellular compartments or the translocation of the media contained inside the organelle where the nanoparticles are located to the cytoplasm (i.e. lysosomes, mitochondria...) [16]. In addition, there are other factors involved. There have been alternative explanations as to how the nanoparticles are able to negotiate biological membranes, like the study by Xiong et al. [14] describing the formation of VNB while irradiating gold nanoparticles with a $561 \mathrm{~nm}$ pulsed laser, which may disrupt the membrane and aid the passage of the nanostructures as a result of the pores generated by the VNBs. All these studies disclosed the enormous complexity that lies behind PTT.

\section{Light at the End of the Tunnel: Research Advances}

These recent finding represent new challenges for the field and highlight the significant potential of nanoparticle-assisted PTT. There is some research, that has shed light on the series of cellular level events that occur when nanoparticles localized inside specific subcellular compartments react to incoming laser irradiation. What is more, the high specificity of some of these nanoplatforms also grants us with a new tool to further understand the function of certain subcellular components and signaling routes, and how they influence the fate of exposed cells during PTT. In a recent publication, Perez-Hernández et al. [15] 

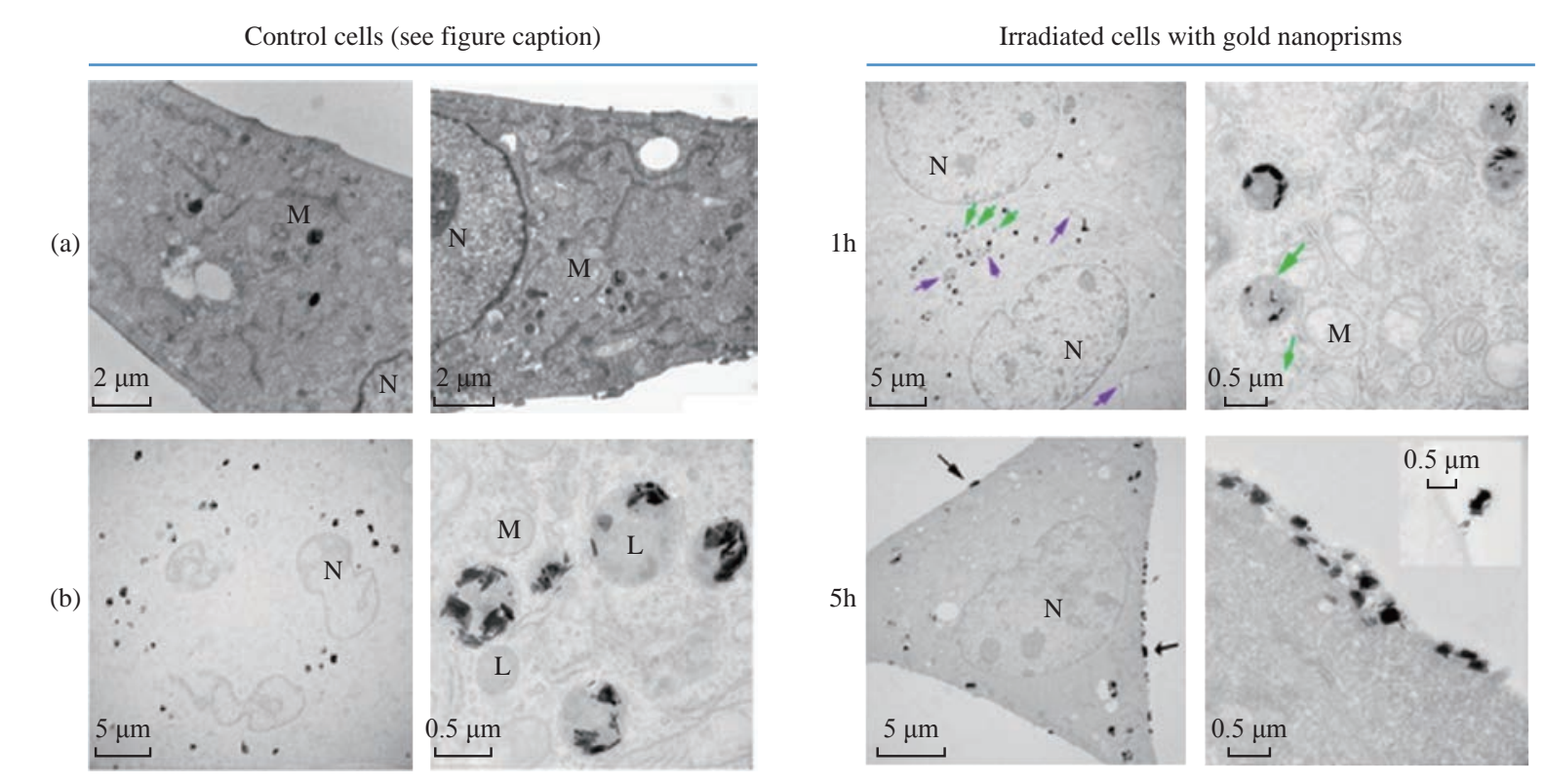

Fig. 1 Localization of NPRs in MEF cells by TEM. Cells were incubated with glucose modified gold nanoprisms (NPRs) overnight and irradiated by a $1064 \mathrm{~nm}$ laser. Cells were then fixed with glutaraldehyde $1 \mathrm{~h}$ and $5 \mathrm{~h}$ after irradiation and prepared for inspection with TEM. Control experiments show NPRs within the lysosomes. One hour after irradiation NPRs can still be observed in the lysosomes (green arrows), but there is a clear presence of the particles in the cytosol (violet arrows). Five hours after irradiation very few NPRs remain in the lysosomes and can be observed embedded within the plasma membrane. (a) Control cells. (b) Control cells without irradiation. N: nucleus. M: mitochondria. L: lysosome. Adapted with permission from Pérez-Hernández et al. [15]. Copyright (C) 2014 American Chemical Society.

were able to specifically induce the apoptotic cell death pathway following PTT using gold nanoprisms localized inside the lysosomes by studying several of the steps involved in this complex signaling pathway. They concluded that the death of the cells was mainly mediated by the mitochondrial signaling pathway of apoptosis, hypothesizing that its activation might come from the cleavage of Bid, a pro-apoptotic protein, by the enzymes released from the lysosome where the nanoparticles are localized.

In addition, a recent study by Aioub and co-workers [12] addressed the issue of the unspecific detrimental effects of ROS derived from PTT. In this study, they synthesized gold nanorods as PTT agents which tips were covered with a platinum shell (PtAuNRs) in an attempt to decrease the ROS generated during the irradiation of the nanoparticles, following the idea that $\mathrm{Pt}$ is able to scavenge the ROS generated during PTT. They compared the above-mentioned PtAuNRs with standard gold nanorods (AuNRs), allowing them to distinguish between the effects of conventional PTT (mediated by AuNRs) and a variation of PTT mediated by a ROS-free action (PtAuNRs). The results were quite clarifying: although AuNRs have a lower heating capability than PtAuNRs, the unprotected gold nanoparticles revealed a higher killing capability, probably caused by the higher levels of ROS measured during the irradiation (Fig. 2). These examples show the increasing interest of the scientific community in starting moving towards the full understanding of PTT.

Finally, Xiong et al. [14] even suggested the possibility to use the VNB they described in order to tune the size of the pores generated on the membrane by changing the intensity of the incoming irradiation. This would allow utilizing a differential diffusion of the target molecules depending on their size. This process may open a wide range of possibilities to be applied in both drug delivery or to see if the release of the content of certain organelles is altering the normal cell functions, which has already being suggested in the past [15].

\section{Conclusion: What Needs to be Done?}

Nanoparticle-mediated PTT is far from simple. It goes without saying that many of the processes that occur during this kind of therapy are still outside our current understanding. It is crucial to independently understand the factors that play a role in PTT before we can deal with such a complex technique. Although there are a few research lines trying to shed light into the processes independently, there is still much more 


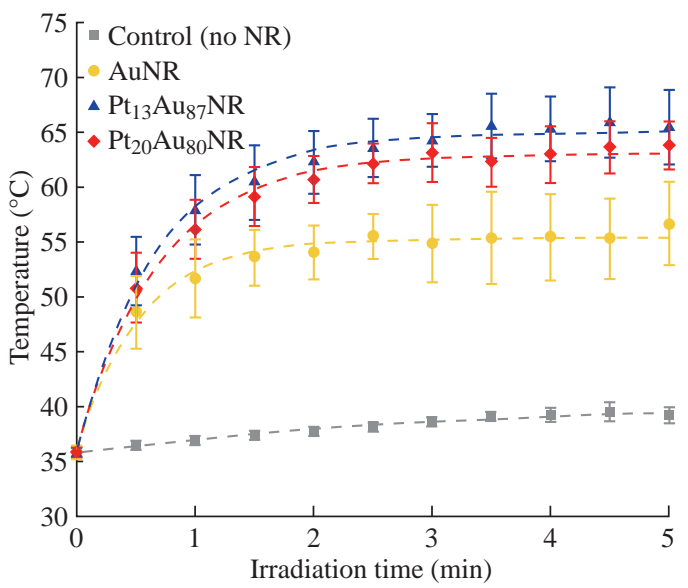

(a)

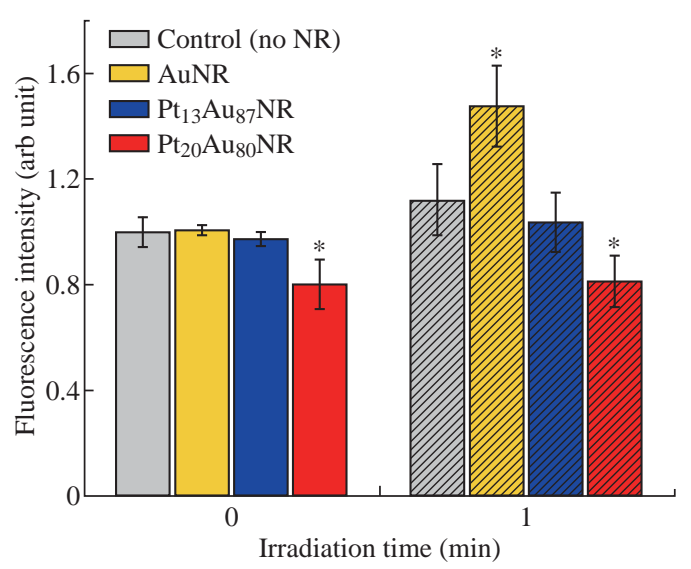

(b)

Fig. 2 (a) Temperature increase induced by PPT heating of cell-free nanorod solutions (all samples with same absorptivity) upon irradiation with $808 \mathrm{~nm}$ laser. Platinum-covered gold nanorods (PtAuNRs) at two different amounts of platinum (blue and red) show the largest temperature increase, higher than standard unprotected AuNRs (yellow). (b) In vitro levels of ROS in cell media before (0 min) and after (1 min) PPT heating. After heating, medium from AuNR-treated cells show significantly higher ROS levels due to heat stress. PtAuNRs do not show significant increases in ROS levels, indicating that the reactive species are reduced before cell damage can occur. Statistical significance $(\mathrm{p}<0.05)$ is indicated by an asterisk. Adapted with permission from Aioub et al. [12]. Copyright $(\mathrm{C}$ 2016 American Chemical Society.

to deal with before PTT can be applied safely and effectively in patients.

One factor is the cellular location of the nanoparticles. The final outcome of the therapy might radically differ if the nanoparticles are located in the interstitial space of the tissue, attached to the plasmatic membrane, or internalized by the cell. Similarly, the effects might change drastically depending on the subcellular location where the nanoparticles would accumulate more. This complexity can only grow if during PTT the membrane of the organelle is compromised and its content is released. In addition, by knowing the different effects that are produced in the different organelles it may theoretically be possible to determine where the nanoparticles are at any given time, as one can distinguish between the different outcomes depending on the subcellular location.

Furthermore, the signaling or metabolic pathways and processes involved during the treatment are key aspects that, depending on this subcellular location, can influence the way the cell reacts to PTT. Different pathways happen in different subcellular locations, and several steps of one single pathway can occur in a different location from one another. A clear example is the different steps of apoptosis, which can occur in a number of subcellular locations from the plasmatic membrane, cytoplasm and mitochondria, and that can be influenced by alterations in other organelles, like lysosomes [15]. This issue just grows more complex when the nanoparticles are able to locate in different organelles at the same time, before, during or after laser irradiation.

Last, but not least, recent research studies have revealed that PTT is much more than mere heating. It is important to understand the effect of the different physicochemical events that occur during PTT in order to prevent undesired effects and/or increase the effectiveness of the treatment. The levels of ROS within the cell can raise either directly caused by irradiation, or indirectly caused by the cell stress mediated by the heat generated [13]. Depending on the nature of the nanoparticle, ROS levels can also rise as a consequence of the release of certain compounds from the nanoparticles themselves during the irradiation [8, 14]. Other effects, like the generation of VNB can also affect the results of the therapy and, if not addressed properly, add another variable to an already complex equation.

To sum up, the full understanding of the heating conditions will allow the treatments to be progressively more precise, effective, and less detrimental to the potential patients. It will ultimately provide us with the ability to choose the best therapeutic design by adjusting the irradiation conditions, subcellular location, dosages, and processes involved. The answer to these questions will eventually strengthen the foundations in many approaches like the understanding of a number of biological processes, the better use of 
multi-modal combined therapies, or a more controlled drug delivery.

\section{Acknowledgements}

This work was supported by Shanghai Jiao Tong University, the Scholarship Council (CSC) grants of the Ministry of Education People's Republic of China; support also came from Fondo Social de la DGA (grupos DGA), Ministerio de la Economía y Competitividad del Gobierno de España for the public funding of Proyectos I+D+I - Programa Estatal de Investigación, Desarrollo e Innovación Orientada a los Retos de la Sociedad (project n. SAF2014-54763-C2-2-R) and ERCPoC HOTFLOW (project 754609). Also, the authors want to thank Scott G. Mitchell for his kind corrections and valuable suggestions.

\section{References}

[1] D. de Melo-Diogo, C. Pais-Silva, D.R. Dias, et al., Strategies to improve cancer photothermal therapy mediated by nanomaterials. Adv. Healthc. Mater., 2017: 1700073.

[2] M.P. Stewart, A. Sharei, X. Ding, et al., Break and enter: in vitro and ex vivo strategies for intracellular delivery. Nature, 2016, 538: 183-192.

[3] R.R. Castillo, M. Colilla, and M. Vallet-Regí, Advances in mesoporous silica-based nanocarriers for co-delivery and combination therapy against cancer. Expert Opin. Drug Deliv., 2017, 14: 229-243.

[4] B. Liu, C. Li, Z. Cheng, et al., Functional nanomaterials for near-infrared-triggered cancer therapy. Biomater. Sci., 2016, 4: 890-909.

[5] W. Qin, G. Huang, Z. Chen, et al., Nanomaterials in targeting cancer stem cells for cancer therapy. Front. Pharmacol., 2017, 8: 1.

[6] Y. Oh, J.O. Jin, and J. Oh, Photothermal-triggered control of sub-cellular drug accumulation using doxorubicinloaded single-walled carbon nanotubes for the effective killing of human breast cancer cells. Nanotechnology, 2017, 28: 125101.

[7] H. Wang, R. Zhao, Y. Li, et al., Aspect ratios of gold nanoshell capsules mediated melanoma ablation by synergistic photothermal therapy and chemotherapy. Nanomedicine, 2016, 12: 439-448.

[8] 8. L. Li, R.H. Leila, M. Yao, et al., CuS nanoagents for photodynamic and photothermal therapies: phenomena and possible mechanisms. Photodiagnosis Photodyn. Ther., 2017, 19: 5-14.

[9] C. Bao, N. Beziere, P. del Pino, et al., Gold nanoprisms as optoacoustic signal nanoamplifiers for in vivo bioimaging of gastrointestinal cancers. Small, 2013, 9: 68-74.

[10] P. Huang, P. Rong, J. Lin, et al., Triphase interface synthesis of plasmonic gold bellflowers as near-infrared light mediated acoustic and thermal theranostics. J. Am. Chem. Soc., 2014, 136: 8307-8313.

[11] 11. J. Zhang, F. Xia, Y. Yang, et al., Human CIK cells loaded with gold nanoprisms as theranostic platform for targeted photoacoustic imaging and enhanced immunophotothermal combined therapy. Nano Biomed. Eng., 2016, 8: 109-124.

[12] 12. M. Aioub, S.R. Panikkanvalappil, and M.A. El-Sayed, Platinum-coated gold nanorods: Efficient reactive oxygen scavengers that prevent oxidative damage toward healthy, untreated cells during plasmonic photothermal therapy. ACS Nano, 2016, 11: 579-586.

[13] L. Minai, D. Yeheskely-Hayon, and D. Yelin, High levels of reactive oxygen species in gold nanoparticle-targeted cancer cells following femtosecond pulse irradiation. Sci. Rep., 2013, 3: 2146.

[14] R. Xiong, K. Raemdonck, K. Peynshaert, et al., Comparison of gold nanoparticle mediated photoporation: Vapor nanobubbles outperform direct heating for delivering macromolecules in live cells. ACS Nano, 2014, 8: 6288-6296.

[15] M. Pérez-Hernández, P. del Pino, S.G. Mitchell, et al., Dissecting the molecular mechanism of apoptosis during photothermal therapy using gold nanoprisms. ACS Nano, 2015, 9: 52-61.

[16] J. Kim, J. Kim, C. Jeong, and W.J. Kim, Synergistic nanomedicine by combined gene and photothermal therapy. Advanced Drug Delivery Reviews, 2016, 98: 99112.

Copyright@ 2017 Gabriel Alfranca, Daxiang Cui, and Jesus Martinez de la Fuente. This is an open-access article distributed under the terms of the Creative Commons Attribution License, which permits unrestricted use, distribution, and reproduction in any medium, provided the original author and source are credited. 